\title{
Advance Feature Extraction Technique for Digital Camera Identification
}

\author{
Radnyeesha R. \\ Sadaphule \\ Upnagr, Nashik road, Nashik, \\ Maharashtra.
}

\author{
Shital R. Kopulkar \\ Jail road, nashik road, Nashik, \\ Maharashtra.
}

\author{
L. K. Chouthmol \\ Kamatwadi, Nashik, \\ Maharashtra
}

\begin{abstract}
On the World Wide Web, a large amount of image content is distributed, owing to the rapid progress in digital camera technologies. For presenting evidence in a court and helping police investigations Digital camera identification, which is the identification of the source camera of input image is becoming increasingly important. A digital camera identification method using the image sensor's pattern noise has received considerable attention in recent years. The existence of differences between the sensitivities of pixels Photo-response nonuniformity (PRNU) noise is mainly generated and it is useful as a fingerprint of a camera. By image processing engine, however the PRNU noise of an image is usually contaminated by random noise and scene content affected by the image processing engine, which inhibits stable
\end{abstract}

Identification. The pairwise magnitude relations of image sensor noise, we proposed a novel digital camera identification method using, which are robust to noise contamination. That the proposed method can identify the source cameras of query images with high accuracy we demonstrate, by performing experiments.

\section{General Terms}

Digital camera identification, PRNU noise, the magnitude relations of clustered PRNU noise.

\section{INTRODUCTION}

As a piece of evidence in a court and identifying the camera that took an image is becoming important as a deterrence to illegal uploads, as a large amount of image content is distributed on the World Wide Web. Camera identification methods using photo-response non-uniformity (PRNU) noise, which is an unique stochastic characteristic of image sensors have been received considerable attenuation. The fingerprint of cameras was proposed is the first approach for camera identification, for this approach was enhanced to improve the identification accuracy. Based on brute-force search for scaled and/or cropped images this approach was enhanced. Moreover, this approach has many applications: for detecting Manuscript received April 4, 2013; revised July 4, 2013 and September 25, 2013; accepted September 25, 2013. Date of publication October 7, 2013; date of current version November 11, 2013. The review of this manuscript and approving it for publication was Prof. Mauro Barni the associate editor coordinating. The authors are with the Department of Electrical and Electronic Engineering, Tokyo University of Agriculture and Technology, Tokyo 184-8588, Japan (e-mail: ytomioka@cc.tuat.ac.jp; 50012645204@st.tuat.ac.jp;kitazawa@cc.tuat.ac.jp). Color versions of one or more of the figures in this paper are available online at http://ieeexplore.ieee.org. Digital Object Identifier10.1109/TIFS.2013.2284761.Forger, classifying scanners, and defending ggainst fingerprint-copy attack. According to the correlation between the PRNU noise of a tested camera and the noise of the query image the source camera of a query image is determined based on camera identification approach basically.

A specified false acceptance rate (FAR) can be achieved, a threshold is determined from the distribution various manufacturers is experimentally estimated the distribution of

Correlations for images taken by different cameras. To obtain the required false acceptance rate a correlation threshold is determined for the camera identification.

This identification is affected by noise contamination caused by the image processing engines of cameras and scene content such as textures on images. The weak components are amplified and contaminated noise so that the strong components are attenuated the proposed method is enhances, in order to relax the effects of scene content. On the basis of texture complexity of each image the method proposed in excludes the noise related to the scene content. Accuracy by Restoring the PRNU noise with a Wiener filter moreover, this method further improves the identification accuracy. However, the identification accuracy is still not sufficient. By cameras of the same model/manufacturer and that have a higher probability of the specified FAR another important issue is that the above camera identification methods may falsely accept images that have been taken. As JPEG compression and color interpolation associated with the sensor design and common image processing this is because of non-unique artifacts (NUAs).

The strength of the effects of NUAs depends on the scenes in the input images, to be higher compared to that for images taken by different cameras the correlation for images taken by cameras of the same model/manufacturer tends. By considering these facts the identification threshold should be determined.

For robust to noise contamination based on the pairwise relationships of pixel clusters, we propose a novel camera identification method for in this paper. We cluster pixels according to the PRNU noise value of a tested camera, in order to reduce the effects of noise contamination in this method. As features robust to disturbances in digital watermarking, pairwise magnitude relations are often used. By pairing clusters with a negative PRNU noise value we consider these features for camera identification, and we generate cluster-pairs and those with a positive PRNU noise value. Because of the PRNU noise, for images taken by the same camera is higher than that for images taken by different cameras our method is based on the fact that the probability of the pairwise Magnitude relation of different cluster-pairs being identical. We determine if a query image has been taken by a tested camera, on the basis of the number of cluster-pairs whose magnitude relation is changed. We divided images taken by different cameras into four image sets according to camera model and image type. In order to determine 
A strict threshold for camera identification with considering NUAs. From the thresholds obtained from those image sets we selected the strictest threshold.

\section{PROPOSED SYSTEM}

\subsection{Problem Statement}

we propose a novel camera identification method based on the pairwise relationships of pixel clusters, which are robust to noise contamination. In this method, in order to reduce the effects of noise contamination, we cluster pixels according to the PRNU noise value of a tested camera. In digital watermarking, pairwise magnitude relations are often used as features robust to disturbances. We consider these features for camera identification, and we generate cluster-pairs by pairing clusters with a negative PRNU noise value and those with a positive PRNU noise value.

\subsection{Goals and Objectives}

Another important issue is that the above camera identification methods may falsely accept images that have been taken by cameras of the same model/manufacturer and that have a higher probability of the specified FAR. This is because of non-unique artifacts (NUAs) associated with the sensor design and common image processing such as JPEG compression and color interpolation. The correlation for images taken by cameras of the same model/manufacturer tends to be higher compared to that for images taken by different cameras, and the strength of the effects of NUAs depends on the scenes in the input images. The identification threshold should be determined by considering these facts.

\subsection{Extraction of PRNU Noise}

An image taken by a digital camera includes noise generated by the image sensor. The noise consists of random noise and photo-response non-uniformity (PRNU) noise. Random noise appears randomly in every image, and it cannot be used for camera identification. In contrast, the PRNU noise is a deterministic component, and it is approximately the same in all images taken by the same camera. The PRNU noise is mainly generated by differences between the sensitivities of pixels. It is unique even across cameras of the same model and is useful as the fingerprint of a camera.

\subsection{Clustered PRNU Noise}

Let ck be a cluster of pixels p1, p2, ..., pn, of amera $\mathrm{C}$, having similar PRNU noise values. The average of the PRNU noise of $\mathrm{UC}(\mathrm{pi})$ in ck is denoted by $\mathrm{U}^{-} \mathrm{C}(\mathrm{ck})$. The first term is the average PRNU noise. The second term is the average random noise. We assume that RI (pi ) is zero mean distribution. Under this assumption, the second term approaches zero as the cluster size increases. The third term is the average noise contamination and could be small. Although $\mathrm{N}^{-} \mathrm{I}$ (ck ) includes small error arising from the third term, we can emphasize its PRNU component by averaging noise residuals.

\subsection{Pixel Clustering Method}

First, pixels are sorted based on the PRNU noise value as in [14]. Let $p 1, p 2, \ldots$, pmp be a sequence of pixels sorted in ascending order of the PRNU noise value in reference pattern $\mathrm{KC}$; $\mathrm{mp}$ is the total number of pixels in $\mathrm{KC}$. For a given cluster size $\mathrm{S}$, the sequence is equally divided into subsequences $\mathrm{c} 1, \mathrm{c} 2, \ldots, \mathrm{cmc} . \mathrm{mc}$ is the number of generated clusters, and it is calculated by $\mathrm{mc}=\mathrm{mp} \mathrm{S}_{\text {. }}$. Each subsequence ck corresponds to a pixel cluster. Fig. 5 shows the distribution of the PRNU noise value in a reference pattern and an example of pixel clusters. For each tested camera C, pixel clusters are generated from its reference pattern $\mathrm{KC}$. When we determine whether an input image has been taken by $\mathrm{C}$, we use pixel clusters generated from KC. From the pixel clusters ck $(1 \leq \mathrm{k} \leq \mathrm{mc})$ of a tested camera $\mathrm{C}$, the clustered PRNU noise $\mathrm{U}^{-} \mathrm{C}(\mathrm{ck})(1 \leq \mathrm{k} \leq \mathrm{mc})$ is calculated. Similarly, $\mathrm{N}^{-} \mathrm{I}(\mathrm{ck})(1 \leq \mathrm{k} \leq \mathrm{mc})$ is calculated for each $\mathrm{I}$.

\section{REFERENCES}

[1] Y. Tomioka and H. Kitazawa, "Digital camera identification based on the clustered pattern noise of image sensors," in Proc. IEEE ICME, Jul. 2011, pp. 1-4.

[2] J. Lukáš, J. Fridrich, and M. Goljan, "Determining digital image origin using sensor imperfections," Proc. SPIE, vol. 5685, pp. 249-260, Apr. 2005.

[3] J. Lukáš, J. Fridrich, and M. Goljan, "Digital "bullet scratches' for images," in Proc. IEEE ICIP, vol. 3. Sep. 2005, pp. 65-68.

[4] J. Lukáš, J. Fridrich, and M. Goljan, "Digital camera identification from sensor pattern noise," IEEE Trans. Inf. Forensics Security, vol. 1, no. 2, pp. 205-214, Jun. 2006.

[5] M. Chen, J. Fridrich, M. Goljan, and J. Lukáš, "Determining image origin and integrity using sensor noise," IEEE Trans. Inf. Forensics Security, vol. 3, no. 1, pp. 74-90, Mar. 2008.

[6] Y. Sutcu, S. Bayram, H. Sencar, and N. Memon, "Improvements on sensor noise based source camera identification," in Proc. IEEE Int. Conf. Multimedia Expo, Jul. 2007, pp. 24-27.

[7] C.-T. Li, "Source camera identification using enhanced sensor pattern noise," in Proc. 16th IEEE Int. Conf. Image Process., Aug. 2009, pp. 7-11.

[8] K. Matsushita and H. Kitazawa, "An improved camera identification method based on the texture complexity and the image restoration," Int. J. Hybrid Inf. Technol., vol. 3, no. 1, pp. 74-90, Jan. 2010.

[9] M. Goljan and J. Fridrich, "Camera identification from cropped and scaled images," Proc. SPIE, vol. 6819, pp. 68190E-1-68190E-13, Mar. 2008. 